\title{
Progressive Postnatal Assembly of Limbic-Autonomic Circuits Revealed by Central Transneuronal Transport of Pseudorabies Virus
}

\author{
Linda Rinaman, ${ }^{1}$ Pat Levitt, ${ }^{2}$ and J. Patrick Card ${ }^{1}$ \\ ${ }^{1}$ Department of Neuroscience, University of Pittsburgh, Pittsburgh, Pennsylvania 15260, and 2Department of \\ Neurobiology, University of Pittsburgh School of Medicine, Pittsburgh, Pennsylvania 15260
}

The development of neuronal projections to a target and the establishment of synaptic connections with that target can be temporally distinct events, which typically are distinguished by functional assessments. We have applied a novel neuroanatomical approach to characterize the development of limbic forebrain synaptic inputs to autonomic neurons in neonatal rats. Transneuronal labeling of preautonomic forebrain neurons was achieved by inoculating the ventral stomach wall with pseudorabies virus (PRV) on postnatal day 1 (P1), P4, or P8. In each age group, PRV-positive neurons were present in autonomic and preautonomic regions of the spinal cord and brainstem 62-64 hr after inoculation. Transneuronal forebrain labeling in rats injected on P8 was similar to the transneuronal labeling reported previously in adult rats and included neurons in the medial and lateral hypothalamus, amygdala, bed nucleus of the stria terminalis, and visceral cortices. However, no cortex labeling and only modest amygdala and bed nucleus labeling were observed in rats injected with PRV on P4, and only medial hypothalamic labeling was observed in rats injected on P1. Additional tracing experiments involving central injections of PRV or cholera toxin $\beta$ indicated that lateral hypothalamic and telencephalic regions projected to the medullary dorsal vagal complex several days before establishing synaptic connections with gastric-related autonomic neurons. These results demonstrate a novel strategy for evaluating synaptic connectivity in developing neural circuits and show a temporally segregated postnatal emergence of medial hypothalamic, lateral hypothalamic, and telencephalic synaptic inputs to central autonomic neurons.

Key words: development; sympathetic; parasympathetic; lateral hypothalamic area; paraventricular nucleus of the hypothalamus; central nucleus of the amygdala; bed nucleus of the stria terminalis; insular cortex; medial prefrontal cortex; dorsal motor nucleus of the vagus; nucleus of the solitary tract; stomach; gastrointestinal
Integrated visceral responses to cognitive or emotional stimuli depend on direct and relayed neural projections from telencephalic and diencephalic components of the limbic forebrain to medullary and spinal components of the autonomic nervous system (LeDoux et al., 1988; Jordan, 1990; Loewy and Spyer, 1990). Little is known about the developmental assembly of these limbic-autonomic circuits. However, their structural and functional maturation likely continues after birth in rats, in which the first postnatal week of development is characterized by a significant amount of synapse formation in the medullary dorsal vagal complex (DVC) (Miller et al., 1983; Rao et al., 1999). The DVC, comprising the parasympathetic dorsal motor nucleus of the vagus and the viscerosensory nucleus of the solitary tract, is the principal autonomic center of the caudal brainstem. DVC neurons receive synaptic inputs from visceral sensory afferents (Shapiro and Miselis, 1985; Rinaman et al., 1989) in addition to prominent inputs from the hypothalamus, amygdala, bed nucleus of the stria terminalis, and visceral cortex (Saper et al., 1976; Van der Kooy et al., 1984; Gray and Magnuson, 1987). Because

Received Dec. 17, 1999; revised Jan. 21, 2000; accepted Jan. 24, 2000.

This work was supported by the National Institute of Mental Health Grant MH01208 and by a research grant from the John T. and Catherine D. MacArthur Foundation Network on Early Experience and Brain Development. James Pacelli and Matthew Roesch contributed importantly to early phases of this study during undergraduate thesis work with L.R. at the University of Pittsburgh.

Correspondence should be addressed to Dr. Linda Rinaman, University of Pittsburgh, Department of Neuroscience, 446 Crawford Hall, Pittsburgh, PA 15260. E-mail: Rinaman@bns.pitt.edu.

Copyright (C) 2000 Society for Neuroscience $\quad 0270-6474 / 00 / 202731-11 \$ 15.00 / 0$ visceral sensory-motor circuits in the DVC already are morphologically well established in newborn rats (Rinaman and Levitt, 1993), the marked postnatal increases in DVC synaptic density could reflect significant postnatal maturation of limbic forebrain inputs to this autonomic center. Similar analyses of synapse formation in spinal autonomic nuclei have not been reported. However, results from tract-tracing studies in developing rats indicate that the earliest forebrain projections to reach thoracic levels of the spinal cord originate from the hypothalamus and only arrive there at approximately the time of birth (Lakke and Hinderink, 1989; Lakke, 1997).

The present study sought to characterize the early postnatal development of limbic forebrain projections to central autonomic neurons in rats. A major goal of these experiments was to distinguish between axonal ingrowth and synapse formation. Such a distinction is critical for determining the connectivity of developing neural circuits and is made possible in the present study by the use of the pseudorabies virus (PRV) transneuronal-tracing method. PRV is a neurotropic $\alpha$-herpesvirus used for the anatomical tracing of synaptically linked neural circuits after peripheral or central inoculation (for review, see Card, 1995, 1998; Enquist and Card, 1996; Enquist et al., 1999). Transport of the attenuated Bartha strain of PRV between CNS neurons occurs only at points of synaptic contact and proceeds in the retrograde direction (i.e., from infected neurons through to their presynaptic afferents). Highly reproducible patterns of first-, second-, and third-order retrograde transynaptic neuronal infection in the spinal cord, medulla, midbrain, diencephalon, and telencephalon 
have been reported in adult rats after PRV inoculation of various visceral targets (cf. Enquist et al., 1999). Here we present new evidence of progressive postnatal synaptic assembly of limbicautonomic circuits, based on age-related increases in the transneuronal labeling of forebrain neurons after inoculation of PRV into the ventral stomach wall in neonatal rats. Additional experiments involved the microinjection of PRV or a standard retrograde tracer directly into the DVC in 1- and 6-d-old rats. Our results provide evidence of a temporally segregated emergence of medial hypothalamic, lateral hypothalamic, and telencephalic synaptic inputs to central autonomic neurons in rats during the first $10 \mathrm{~d}$ of postnatal development.

\section{MATERIALS AND METHODS}

Animals. Experimental protocols were approved by the University of Pittsburgh Institutional Animal Care and Use Committee and conformed to regulations detailed in the National Institutes of Health publication Guide for the Care and Use of Laboratory Animals. Pregnant multiparous Sprague Dawley rats were purchased from Zivic-Miller (Zelienople, PA). Rats were housed in a standardized environment $\left(20-22^{\circ} \mathrm{C} ; 12 \mathrm{hr}\right.$ of light beginning at 7 A.M.) in individual polyethylene tubs filled with Sanicel bedding and were given water and pelleted rat chow (Purina) ad libitum. Cages were checked mornings and evenings for newborn pups. The day of birth was designated postnatal day 0 (P0). Litters were culled to 10 pups on $\mathrm{P} 0$ or P1. Both male and female pups were used.

Virus. The Bartha strain of PRV [PRV-Bartha (Bartha, 1961)] was used in this study. The virus, grown to a titer of $2.0 \times 10^{8} \mathrm{pfu} / \mathrm{ml}$, was provided by Dr. Lynn Enquist (Princeton University, Princeton, NJ). PRV-Bartha is an attenuated vaccine strain with well defined alterations of the viral genome that reduce virulence. Details regarding the preparation, characterization, genetics, handling, and experimental use of PRV-Bartha for transneuronal tracing have been reported (Card and Enquist, 1995; Enquist and Card, 1996). All experimental manipulations involving PRV were conducted in a laboratory approved for Biosafety Level 2 studies.

Stomach wall inoculation. Neonatal rats were anesthetized on P1, P4, or P8 ( $n \geq 10$ per group) by ice-induced hypothermia. A short scalpel incision was made through the skin to expose the translucent abdominal muscle layers and the underlying white, milk-filled stomach. With the aid of a surgical microscope, the sharpened tip of a $5 \mu$ l Hamilton syringe (outer diameter, $0.47 \mathrm{~mm}$ ) containing PRV was inserted tangentially through the abdominal muscle layers and peritoneal membrane into the ventral stomach wall. A total volume of $1.0 \mu \mathrm{l}$ of PRV was injected at two to three sites. Control rats (P8; $n=3)$ were injected with the same volume of PRV delivered into the abdominal muscles overlying the stomach, instead of into the stomach wall. [In a previous study, to control for the effects of the possible nonspecific peripheral spread of PRV from stomach wall inoculation sites, the same amount of PRV was injected either intragastrically (intraluminally) or intraperitoneally in 1-d-old rats (Rinaman et al., 1999). The results indicated that PRV injected into the ventral stomach wall in neonatal rats did not produce any central labeling that was attributable to leakage from the injection site.] After PRV inoculation, the syringe was removed, and the abdominal muscle layer was rinsed with saline and blotted dry. The skin incision was closed with Ethilon 6-0 silk sutures. Pups were warmed in an incubator $\left(34^{\circ} \mathrm{C} ; 60 \%\right.$ humidity) until they regained full recovery of respiration and mobility and then were returned to their dams and home cage.

Injected pups were inspected several times daily to ensure that they remained asymptomatic and received adequate maternal care during the postinoculation period. A $62-64 \mathrm{hr}$ survival time after stomach wall inoculation was used for all rats included in this study. Preliminary experiments demonstrated that this survival time was sufficient to achieve consistent retrograde transneuronal infection of limbic forebrain neurons in the largest rats used in this study, i.e., those injected with PRV on P8 and killed on P10.5 (see Results). We reasoned that a 62-64 hr survival time also would be sufficient for potential retrograde transneuronal transport of PRV to the limbic forebrain in younger (smaller) rat pups, provided that the central synaptic connections necessary for such transport were present. Longer postinoculation survival times were not used in this study, because many rat pups injected with PRV into the stomach wall on P1 began to display adverse symptoms of infection (e.g., apparent dehydration, pallor, absence of visible milk in the stomach, and maternal exclusion from the nest) $\sim 60 \mathrm{hr}$ after inoculation. Rat pups that displayed adverse symptoms before $62 \mathrm{hr}$ after inoculation were immediately anesthetized and killed and were not included in the present study.

Dorsal vagal complex injection. A second set of experiments was performed to determine (1) the extent to which axons of diencephalic and telencephalic neurons project to the vicinity of the DVC in neonatal rats and (2) whether such projection neurons are capable of PRV uptake, transport, and replication. For this purpose, either PRV or a standard retrograde tracer [cholera toxin $\beta$ subunit $(\mathrm{CTb})$; List Biologic, Campbell, CA] was injected directly into the DVC in rats on P1 ( $n \geq 7$ for each tracer). Additional rats were injected with CTb into the DVC on P6 $(n=$ 6). Rats were anesthetized by hypothermia and placed into a machined stereotaxic adaptor (Stoelting) designed for use in neonatal rats (Cunningham and McKay, 1993). A short longitudinal scalpel incision was made through the skin overlying the occipital ridge. With the aid of a surgical microscope, dorsal neck muscles were bluntly dissected and retracted to reveal the roof of the fourth ventricle. The ventricular space was opened with a 25 gauge needle to visualize the dorsal surface of the caudal medulla and the area postrema (AP). A sharp, beveled glass micropipette (75-100 $\mu \mathrm{m}$ outer tip diameter) was filled with either PRV $\left(1 \times 10^{8} \mathrm{pfu} / \mathrm{ml}\right)$ or $\mathrm{CTb}(0.25 \%$ in $0.15 \mathrm{M} \mathrm{NaCl})$ and attached to a 1.0 $\mu l$ Hamilton syringe held in a mounted micromanipulator. The tip of the micropipette was positioned on the dorsal medullary surface at the lateral edge of the AP and then lowered $0.1-0.3 \mathrm{~mm}$ into the subjacent DVC. A total volume of $50 \mathrm{nl}$ of either PRV or CTb was manually pressure-injected into the DVC over $\sim 2 \mathrm{~min}$. The syringe was left in place for 2 min after injection and then withdrawn. The skin incision was closed with Ethilon 6-0 nylon sutures. Pups were allowed to recover from hypothermia in an incubator $\left(34^{\circ} \mathrm{C}\right.$; $60 \%$ humidity) and then were returned to their dam and home cage. Pups remained with their dam for $48-50 \mathrm{hr}$ before being anesthetized and killed by perfusion fixation (described below). All CTb-injected pups and most PRV-injected pups remained asymptomatic during this survival interval, although it was necessary to anesthetize and kill some PRV-injected rat pups (not included in this study) that displayed adverse symptoms of infection before $48 \mathrm{hr}$ had elapsed.

Perfusion fixation and tissue preparation. After the appropriate survival interval, rats were deeply anesthetized by intraperitoneal injection of 8.3 $\mathrm{mg}$ of ketamine and $1.7 \mathrm{mg}$ of xylazine (Fort Dodge Labs) and perf used transcardially with $25 \mathrm{ml}$ of $0.15 \mathrm{M} \mathrm{NaCl}$ followed by $75-100 \mathrm{ml}$ of $4 \%$ paraformaldehyde containing $1.4 \%$ L-lysine and $0.2 \%$ sodium metaperiodate (McLean and Nakane, 1974). Fixed brains were removed from the skull, post-fixed at $4^{\circ} \mathrm{C}$ for $12-20 \mathrm{hr}$, and cryoprotected at $4^{\circ} \mathrm{C}$ for at least $24 \mathrm{hr}$ in aqueous $20 \%$ sucrose. The spinal cord from a subset of cases in each experimental group was removed from the vertebral column and treated similarly. Coronal $40-\mu \mathrm{m}$-thick brain sections were cut from the olfactory tubercle to the $\mathrm{C} 2$ level of the spinal cord using a freezing microtome. Thoracic and lumbar segments of the spinal cord were sectioned longitudinally at $50 \mu \mathrm{m}$. Sections were collected serially in four sets and stored at $-20^{\circ} \mathrm{C}$ in cryopreservant (Watson et al., 1986). Before immunocytochemical processing, tissue sections were removed from cryopreservant and rinsed for $1 \mathrm{hr}$ with gentle agitation in several changes of buffer (0.1 M sodium phosphate, $\mathrm{pH} 7.2)$.

Antisera and immunocytochemical methods. Primary and secondary antisera were diluted in buffer containing $1 \%$ normal donkey serum (Jackson ImmunoResearch, West Grove, PA) and 0.3\% Triton X-100 (Sigma, St. Louis, MO). A rabbit polyclonal antiserum (Rb133; provided by Dr. Lynn Enquist, Princeton University) was used to localize PRV in tissue sections. This antiserum recognizes major PRV membrane and capsid proteins (Card et al., 1990). A goat polyclonal antiserum (List Biologic) was used to localize CTb neural tracer.

For immunoperoxidase labeling of either PRV or CTb, tissue sections were incubated at $4^{\circ} \mathrm{C}$ for $48-72 \mathrm{hr}$ in primary antiserum (Rb133 or goat anti-CTb diluted 1:20,000 or 1:50,000, respectively). All subsequent processing was performed at room temperature. Sections were rinsed for $1 \mathrm{hr}$ in several changes of buffer, incubated for $1 \mathrm{hr}$ in affinity-purified biotinylated donkey anti-rabbit or anti-goat IgG (1:500; Jackson ImmunoResearch), rinsed in buffer, and then incubated for $1.5 \mathrm{hr}$ in Vectastain Elite avidin-biotin reagents (Vector Laboratories, Burlingame, CA; $9 \mu \mathrm{l}$ of $A+9 \mu \mathrm{l}$ of $B$ per $1.0 \mathrm{ml}$, combined 90 min before tissue incubation). After buffer rinses, sections were immersed for $5 \mathrm{~min}$ in a solution of $0.05 \%$ diaminobenzidine and $0.01 \% \mathrm{H}_{2} \mathrm{O}_{2}$ to generate a brown immunoperoxidase reaction product. Immunoreacted tissue sections were 

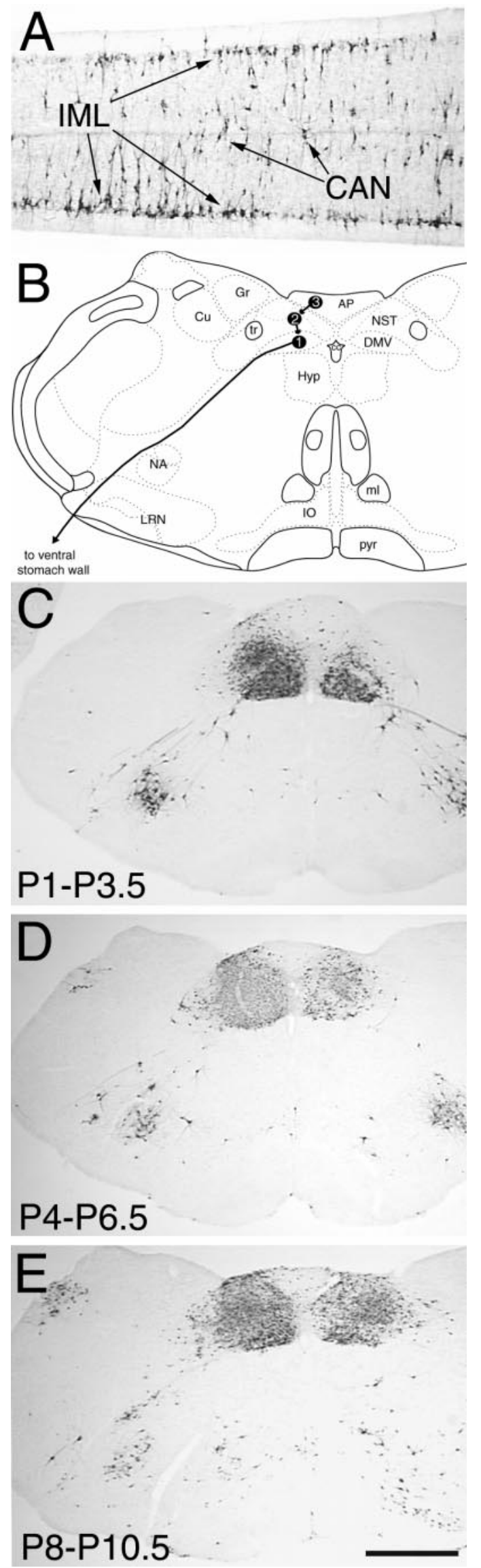

rinsed, mounted onto Superfrost Plus glass slides (Fisher Scientific, Houston, TX), dehydrated in a graded series of ethanol, cleared in xylene, and coverslipped with Cytoseal 60 (VWR). An adjacent set of sections was mounted onto slides and stained for Nissl substance with cresyl violet to clarify the anatomical location of PRV- or CTbimmunopositive cells.

Data analysis. Every fourth section (160 $\mu \mathrm{m}$ frequency) through the brainstem and forebrain was examined for PRV or CTb immunolabeling by the use of a Zeiss Axiophot light microscope equipped with Nomarski optics. Retrogradely labeled cells were easily identified by their cytoplasmic content of dark brown immunoperoxidase reaction product. The distribution of PRV- or CTb-labeled somata in the diencephalon and telencephalon was recorded in each experimental case, with reference as necessary to adjacent Nissl-stained sections to verify anatomical locations. The distribution of labeled forebrain neurons in representative cases was plotted by the use of a computerized data acquisition system (StereoInvestigator) for purposes of illustration.

Quantitative analysis of retrograde transneuronal labeling was performed in rats that received PRV injections into the ventral stomach wall. PRV-immunopositive forebrain neurons were counted bilaterally in every slide-mounted tissue section (160 $\mu \mathrm{m}$ frequency) through the anatomical region of interest. Cell count values were corrected according to the following formula: $N=[T /(T+D)] n$, where $N$ is the corrected cell number, $n$ is the number of cells counted, $T$ is the section thickness (always $40 \mu \mathrm{m}$ in the present study), and $D$ is the mean diameter of counted cells (Abercrombie, 1946). The mean diameter of labeled neurons in each diencephalic and telencephalic region in each age group was determined by measuring the size of 25 representative labeled neurons per region (or by measuring all labeled neurons in the region when $<25$ were present). Corrected counts were combined by age group and expressed as the mean \pm SE. Age-related differences in PRV labeling in each forebrain region were tested for statistical significance by the use of two-way ANOVA. When $f$ values indicated significant effects, the ANOVA was followed up with planned post hoc comparisons using Dunn's (Bonferroni) correction to control for repeated measures analysis. Differences were considered significant when $p<0.05$.

Photomicroscopy and preparation of illustrations. Tissue sections were photographed with Kodak Ektachrome 160T color slide film by the use of a $35 \mathrm{~mm}$ camera mounted onto the microscope. Color slides were developed commercially and then scanned (Polaroid SprintScan 35) into Adobe Photoshop 5.0. Images were converted to black and white and balanced to obtain consistent white levels in nontissue regions (e.g., open blood vessels and ventricular spaces).

\section{RESULTS}

\section{Central PRV transport after stomach wall injection: spinal cord and brainstem labeling}

Data from 19 rats that received stomach wall injections of PRV on $\mathrm{P} 1(n=7), \mathrm{P} 4(n=5)$, or P8 $(n=7)$ and were killed $62-64 \mathrm{hr}$ later (i.e., on P3.5, P6.5, or P10.5, respectively) were analyzed. The distribution and extent of spinal cord and brainstem PRV labeling (described below) were similar in all of these rats, with no consistent differences among age groups.

PRV-positive neurons were observed bilaterally in sympathetic regions of the thoracic and upper lumbar spinal cord (i.e., the

Figure 1. PRV immunolabeling in the thoracolumbar spinal cord $(A)$ and caudal brainstem $(C-E)$ 62-64 hr after inoculation of the ventral stomach wall in rats on $P 1(A, C), P 4(D)$, or $P 8(E)$. B, A schematic diagram of the caudal brainstem [modified from Swanson (1992)] indicating the temporal progression of PRV retrograde transneuronal infection from the stomach wall to preganglionic motor neurons in the $D M V(1)$, to preautonomic neurons in the NST (2), and finally to neurons in the $A P$ (3), as determined in previous work (Rinaman et al., 1999). AP, Area postrema; $C A N$, central autonomic nucleus; $C u$, cuneate nucleus; $D M V$, dorsal motor nucleus of the vagus; $G r$, gracile nucleus; Hyp, hypoglossal motor nucleus; $I M L$, intermediolateral cell column; $I O$, inferior olive; $L R N$, lateral reticular nucleus; $\mathrm{ml}$, medial lemniscus; $N A$, nucleus ambiguus; NST, nucleus of the solitary tract; $P 1$, postnatal day 1; pyr, pyramidal tract; $t r$, solitary tract. Scale bar: $C-E, 1 \mathrm{~mm} ; A, 200 \mu \mathrm{m}$. 


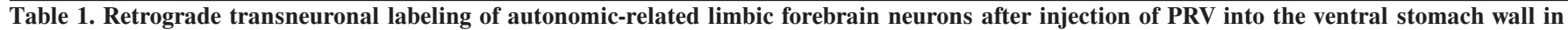
developing rats

\begin{tabular}{|c|c|c|c|c|c|c|c|}
\hline \multirow[b]{2}{*}{ Group $(n)$} & \multicolumn{3}{|c|}{ Diencephalon } & \multicolumn{4}{|c|}{ Telencephalon } \\
\hline & $\mathrm{DMH}$ & LHA & PVN & $\mathrm{CeA}$ & BNST & IC & $\mathrm{mPFC}$ \\
\hline P1-P3.5 (7) & $21 \pm 6$ & 0 & $232 \pm 16$ & $1 \pm 1$ & 0 & 0 & 0 \\
\hline P4-P6.5 (5) & $52 \pm 11^{*}$ & $41 \pm 9^{*}$ & $397 \pm 37^{*}$ & $218 \pm 29^{*}$ & $30 \pm 6^{*}$ & 0 & 0 \\
\hline P8-P10.5 (7) & $62 \pm 10^{*}$ & $135 \pm 16^{* *}$ & $>500^{a}$ & $>500^{a}$ & $139 \pm 25^{* *}$ & $78 \pm 21^{* *}$ & $95 \pm 26^{* *}$ \\
\hline
\end{tabular}

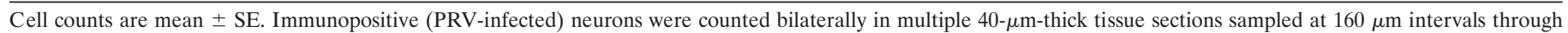

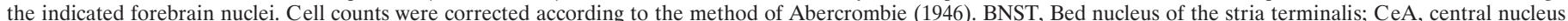

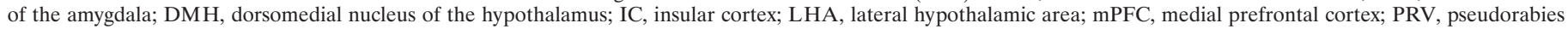
virus; PVN, paraventricular nucleus of the hypothalamus.

${ }^{a}$ Uncorrected cell count value (see Results).

*Significantly greater than the P1-P3.5 group $(p<0.05)$.

**Significantly greater than both the P1-P3.5 and P4-P6.5 groups ( $p<0.05$ for each comparison).

Figure 2. PRV immunolabeling in the diencephalon $62-64 \mathrm{hr}$ after inoculation of the ventral stomach wall in rats on P1. A, A computer-assisted tracing of a single tissue section in which the distribution of all PRV-labeled neurons is plotted. Note that the $C e A$ does not contain any labeled neurons. $B$, Photomicrograph of PRV-positive neurons in the $p f P V N$. $C$, Labeled neurons in the medial (right) and lateral (left) parvocellular subdivisions of the $P V N$. CeA, Central nucleus of the amygdala; $O T$, optic tract; $p f P V N$, perifornical division of the $P V N ; P V N$, paraventricular nucleus of the hypothalamus; III $v$., third ventricle. Scale bars, $200 \mu \mathrm{m}$.
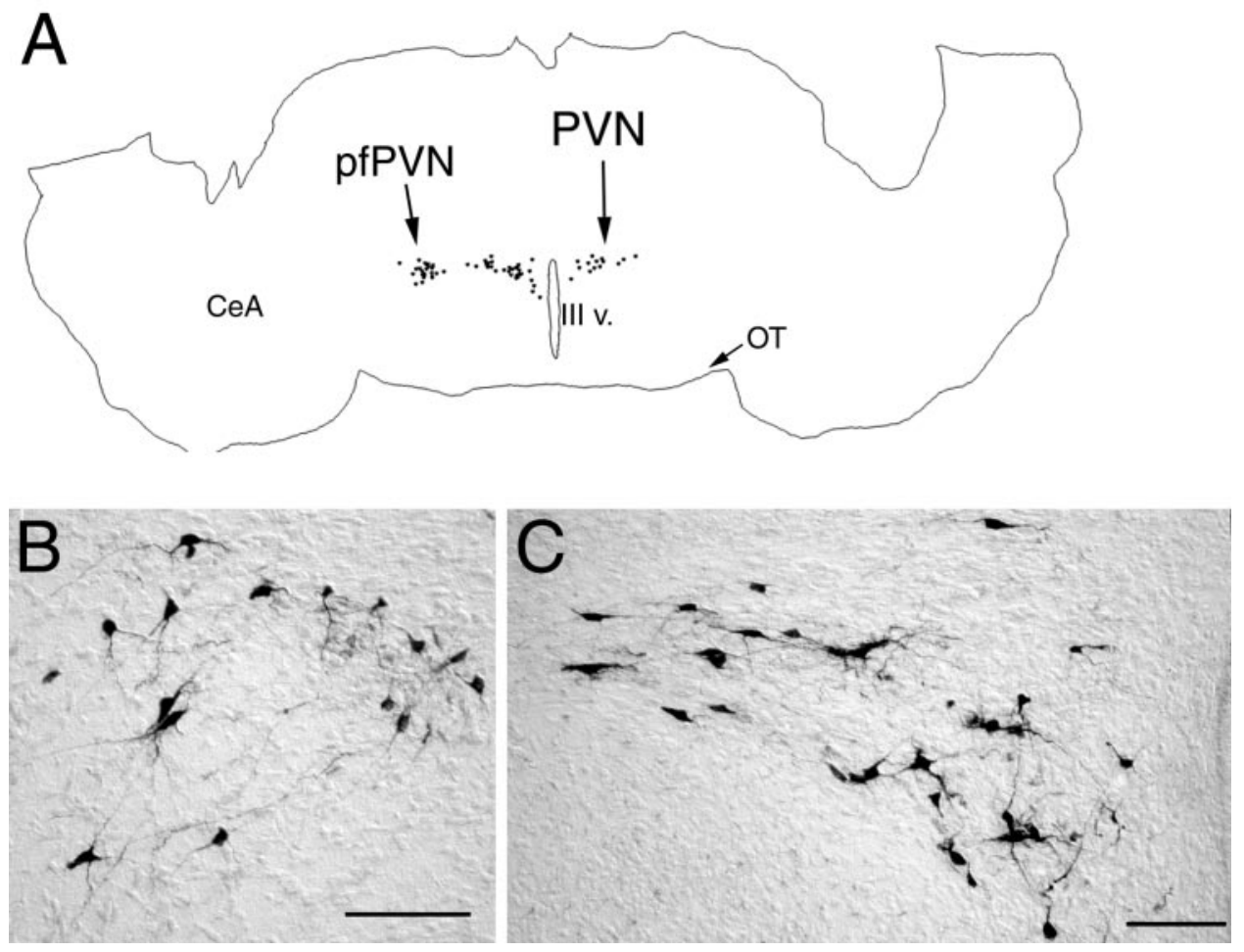

intermediolateral cell column and central autonomic nucleus) and in the parasympathetic DVC in all rats after stomach wall inoculation (Fig. 1). Neural infection in the DVC always was more prominent on the left side, where preganglionic vagal motor neurons that project to the ventral stomach wall are located (Shapiro and Miselis, 1985; Rinaman et al., 1999). As shown schematically in Figure 1, previous time course studies in adult and newborn rats have shown that vagal preganglionic motor neurons are the first DVC neurons to become infected after stomach wall inoculation, followed by neurons in the medial nucleus of the solitary tract (which innervate vagal motor neurons) and then followed by neurons in the AP (which innervate neurons in the nucleus of the solitary tract) (Rinaman et al., 1993, 1999). In all three age groups, transneuronally infected brainstem neurons were present bilaterally in the AP (Fig. 1) and also were present in the caudal raphe (obscurus and pallidus), paramedian reticular nucleus, caudal and rostral ventrolateral medulla (including the $\mathrm{A} 1, \mathrm{C} 1$, and $\mathrm{A} 5$ catecholamine cell group regions), subcoeruleus nucleus, Barrington's nucleus, pedunculopontine tegmental nucleus, and ventrolateral periaqueductal gray (data not shown). These results replicate our previous findings in rats that received $2.0 \mu \mathrm{l}$ of PRV injected into the ventral stomach wall on P1 (Rinaman et al., 1999).

\section{Central PRV transport after stomach wall injection: forebrain labeling}

The distribution and number of transneuronally infected diencephalic and telencephalic neurons increased progressively during postnatal development (Table 1). PRV immunolabeling was observed bilaterally in the dorsomedial nucleus of the hypothalamus $(\mathrm{DMH})$ and in the dorsal, medial, lateral, and perifornical (forniceal) parvocellular subdivisions of the paraventricular nucleus of the hypothalamus (PVN) in all three age groups (Table 1; Figs. 2, 3, 4). Perifornical neurons technically can be considered a component of the lateral hypothalamic area (LHA); indeed, we designated them LHA neurons in a recent report (Rinaman et al., 1999). However, in the present report, labeled perifornical hypothalamic neurons at the level of the PVN were counted together 


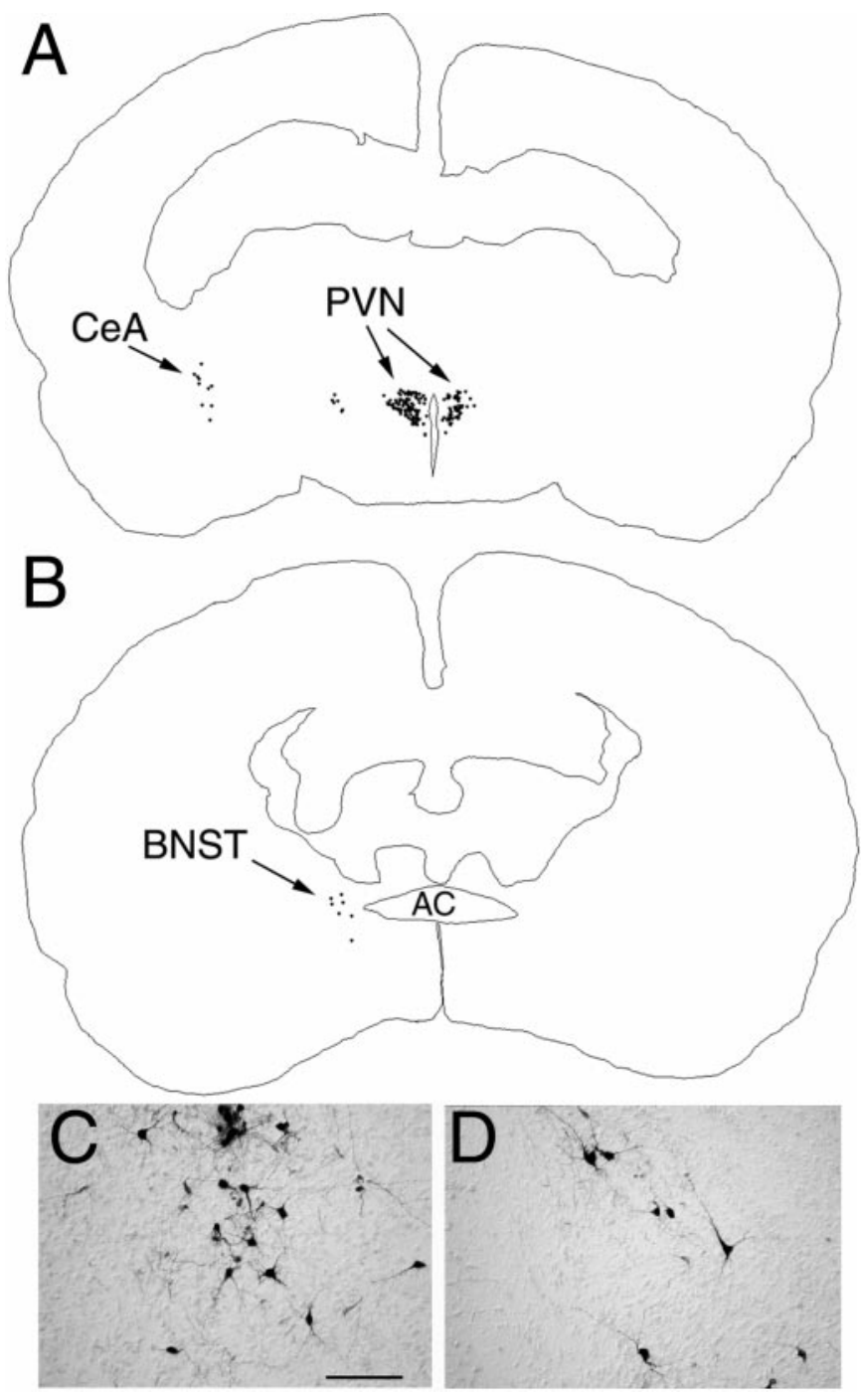

Figure 3. PRV immunolabeling in the diencephalon and telencephalon 62-64 hr after inoculation of the ventral stomach wall in rats on P4. $A, B$, Computer-assisted tracings of single tissue sections in which the distribution of all PRV-labeled neurons is plotted. $C$, Photomicrograph of PRVpositive neurons in the medial $C e A$. $D$, Labeled neurons in the dorsolateral BNST. AC, Anterior commissure; BNST, bed nucleus of the stria terminalis; $C e A$, central nucleus of the amygdala; $P V N$, paraventricular nucleus of the hypothalamus. Scale bar, $200 \mu \mathrm{m}$.

with the PVN, on the basis of their observed anatomical contiguity with the lateral parvocellular subdivision (see Fig. 2). Labeled neurons located caudal to the PVN (as far as the caudal subthalamic nucleus) and lateral to the fornix were counted as LHA neurons in the present report.

Apart from the DMH and PVN labeling noted above, no other forebrain labeling was present in the P1-P3.5 age group. Specifically, no PRV-positive neurons were observed in the caudal LHA, bed nucleus of the stria terminalis (BNST), insular cortex (IC), or infralimbic medial prefrontal cortex (mPFC) in the P1P3.5 group (Table 1). Amygdala labeling also was absent in this age group, except in one case (out of seven examined) in which a total of six PRV-positive neurons were counted in the medial subdivision of the central nucleus of the amygdala (CeA). In contrast, PRV labeling consistently was observed in the caudal LHA (at the level of the subthalamic nucleus), medial CeA, and
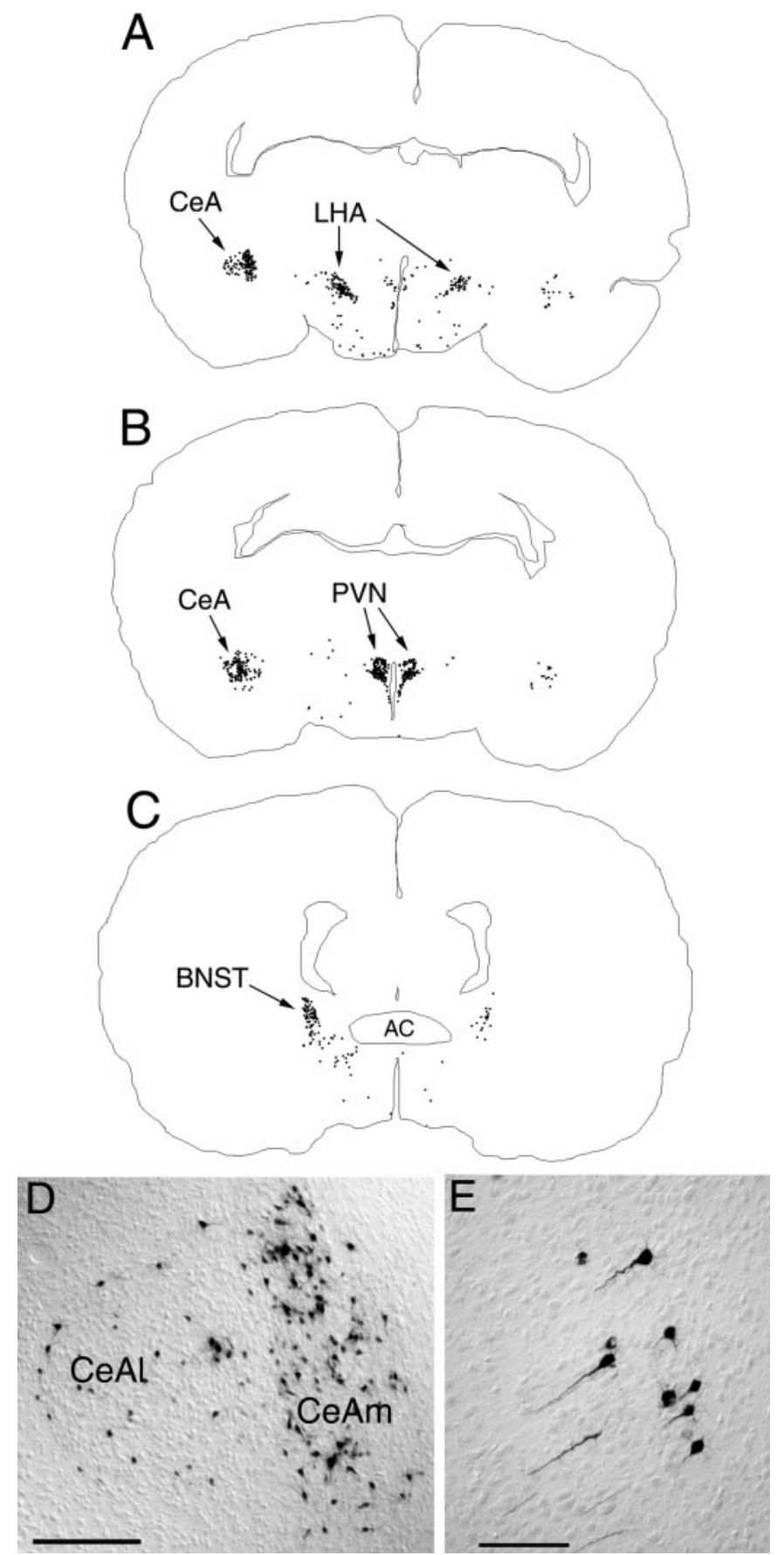

Figure 4. PRV immunolabeling in the diencephalon and telencephalon 62-64 hr after inoculation of the ventral stomach wall in rats on P8. $A-C$, Computer-assisted tracings of single tissue sections in which the distribution of all PRV-labeled neurons is plotted. $D$, Photomicrograph of PRVpositive neurons in the $C e A$. E, Labeled neurons in the insular cortex. $A C$, Anterior commissure; $B N S T$, bed nucleus of the stria terminalis; $C e A$, central nucleus of the amygdala, medial $(\mathrm{m})$ or lateral $(l) ; L H A$, lateral hypothalamic area; $P V N$, paraventricular nucleus of the hypothalamus. Scale bars: $D, 500 \mu \mathrm{m} ; E, 200 \mu \mathrm{m}$.

laterodorsal BNST in the $\mathrm{P} 4-\mathrm{P} 6.5$ and $\mathrm{P} 8-\mathrm{P} 10.5$ age groups (Table 1; Figs. 3, 4).

The overall distribution of diencephalic and telencephalic labeling observed in the P8-P10.5 age group was qualitatively similar to the labeling observed in adult rats after PRV inoculation of the ventral stomach wall, although in adult rats such 
forebrain labeling requires longer postinoculation survival intervals (Rinaman et al., 1993; Yang et al., 1999). The high density of PRV immunolabeling in the PVN and CeA in rats from the P8-P10.5 age group made it difficult to discriminate reliably and count each individual PRV-positive neuron in those brain regions. Therefore, counting was discontinued in the $\mathrm{P} 8-\mathrm{P} 10.5$ age group after 500 distinctly labeled PVN neurons and 500 distinctly labeled $\mathrm{CeA}$ neurons were counted in each experimental case (Table 1). Counts in these two brain regions (Table 1, indicated as $>500$ ) were not corrected with the method of Abercrombie (1946), and it was clear that many labeled PVN and CeA cells went uncounted in each of the seven experimental cases in this age group. Although PRV-positive neurons were absent in the IC and $\mathrm{mPFC}$ in the $\mathrm{P} 4-\mathrm{P} 6.5$ age group, both visceral cortex regions consistently contained many labeled neurons in the P8-P10.5 group (Table 1; Fig. 4).

\section{Central PRV transport after control injections of PRV into abdominal muscles}

A very different pattern of CNS infection was observed in rats that received PRV injections into the ventral abdominal musculature on P8 $(n=3)$. Spinal cord, brainstem, and cerebellar labeling in these rats was consistent with retrograde transneuronal transport of PRV through both sympathetic and somatic neural circuits (Fig. 5). Parasympathetic motor neurons in the DVC were not infected. A small number of infected neurons were observed in the lateral nucleus of the solitary tract, but none were observed in the AP (Fig. 5). Infected forebrain neurons were prominent in the caudal LHA, DMH, dorsal and medial parvocellular PVN, primary motor cortex (layer V pyramidal neurons), and supplemental somatosensory cortex (Fig. 5). Importantly, despite clear infection of spinal and brainstem sympathetic and presympathetic neurons, no infected neurons were observed in the CeA, BNST, IC, or infralimbic mPFC, all regions that did contain infected neurons after injection of PRV into the ventral stomach wall on P8.

\section{Forebrain labeling after direct injection of PRV or CTb into the dorsal vagal complex}

To ascertain whether autonomic-related forebrain neurons are capable of PRV uptake, transport, and replication in neonatal rats, we injected either $\mathrm{PRV}$ or a standard retrograde tracer (CTb) directly into the DVC on P1 or P6. All rats were anesthetized and killed 48-50 hr after tracer injection. The location and size of each injection site was determined by examining PRV or $\mathrm{CTb}$ immunoperoxidase labeling in the caudal brainstem. Rats with injection sites that were appropriately centered in the medial DVC included five rats injected with PRV on $\mathrm{P} 1$, three rats injected with $\mathrm{CTb}$ on $\mathrm{P} 1$, and four rats injected with $\mathrm{CTb}$ on $\mathrm{P} 6$. Injection sites in these cases invariably involved the AP, gracile nucleus, hypoglossal motor nucleus, and/or dorsal reticular formation (Fig. 6).

The distribution and overall extent of retrograde labeling in the forebrain were similar after injection of either PRV or CTb into the DVC on P1, with one notable exception; many more DMH neurons were labeled after PRV injections than were labeled after $\mathrm{CTb}$ injections. In contrast to the results after injection of PRV into the stomach wall on P1, after DVC injection on P1 both tracers produced substantial retrograde labeling in medial, lateral, and perifornical parvocellular subnuclei of the PVN, caudal LHA, medial CeA, and laterodorsal BNST. Retrogradely labeled neurons were not present in the IC or $\mathrm{MPFC}$ after DVC injection

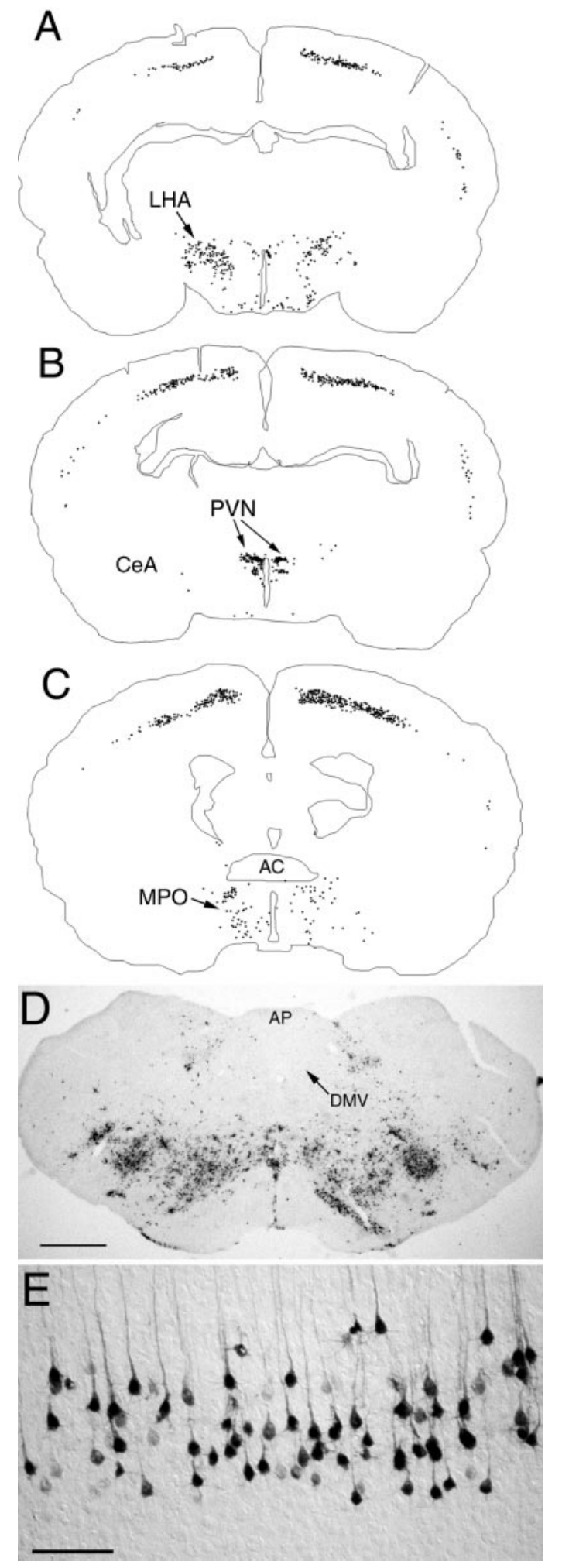

Figure 5. PRV immunolabeling in the diencephalon and telencephalon 62-64 hr after inoculation of the abdominal muscles in control rats on P8. $A-C$, Computer-assisted tracings of single tissue sections in which the distribution of all PRV-labeled neurons is plotted. $D$, Photomicrograph of PRV-positive neurons in the caudal brainstem. $E$, Labeled neurons in the primary motor cortex (layer V). $A C$, Anterior commissure; $A P$, area postrema; $C e A$, central nucleus of the amygdala; $D M V$, dorsal motor nucleus of the vagus; $L H A$, lateral hypothalamic area; $M P O$, medial preoptic area; $P V N$, paraventricular nucleus of the hypothalamus. Scale bars: $D, 500 \mu \mathrm{m} ; E, 200 \mu \mathrm{m}$. 

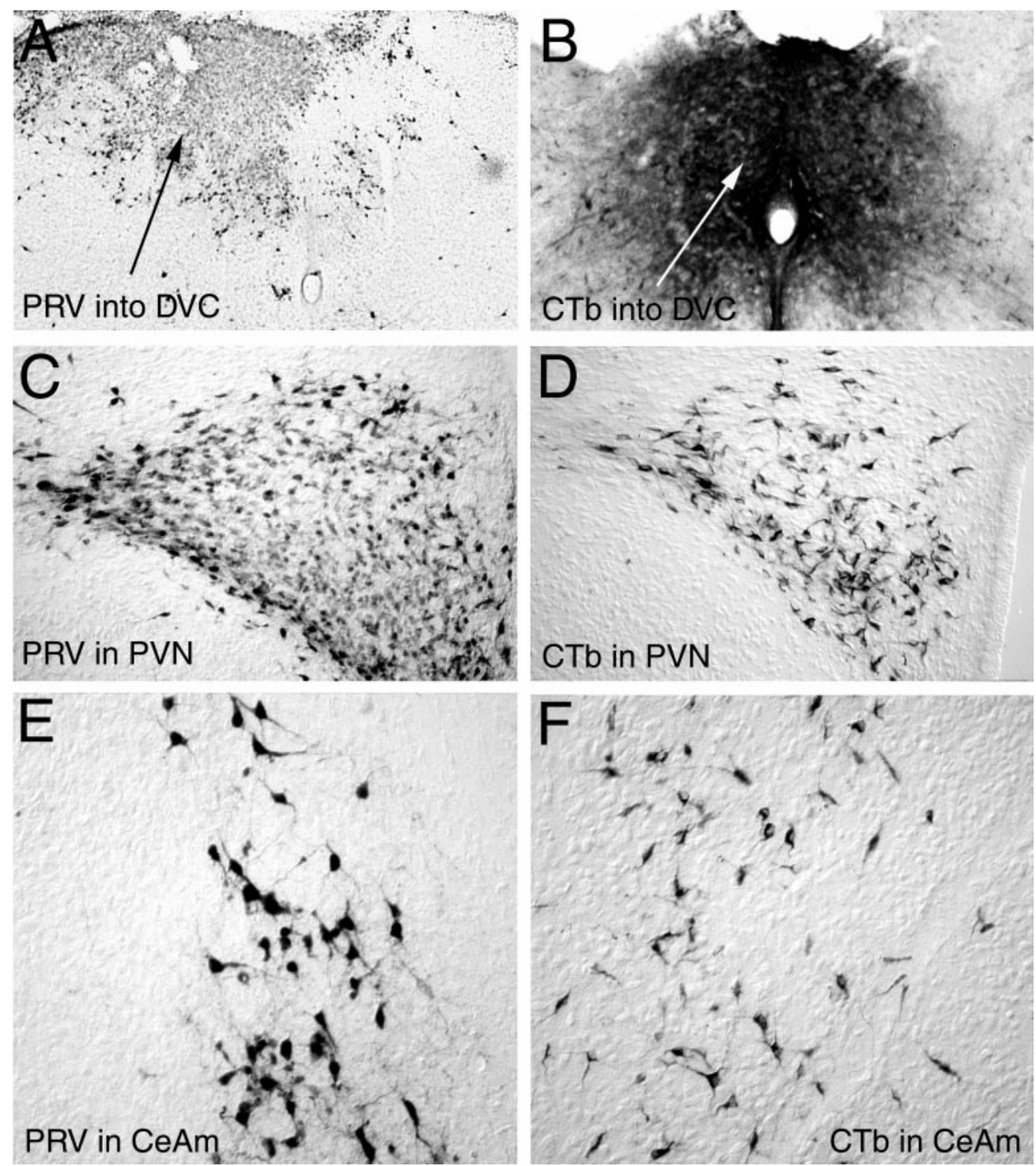

Figure 6. Photomicrographs of $P R V$ and $C T b$ immunolabeling $48-50 \mathrm{hr}$ after injection of either tracer directly into the $D V C$ on P1. $A, B$, Typical $P R V$ $(A)$ or $C T b(B)$ injection sites in the caudal dorsomedial medulla. $C$, $D$, Retrograde $P R V(C)$ or $C T b(D)$ labeling in the $P V N$. E, F, Retrograde $P R V(E)$ or $C T b(F)$ labeling in the medial $C e A$. $C e A$, Central nucleus of the amygdala, medial $(m)$; $C T b$, cholera toxin $\beta$ subunit; $D V C$, dorsal vagal complex; $P R V$, pseudorabies virus; $P V N$, paraventricular nucleus of the hypothalamus. of either tracer on P1. In contrast, injections of $\mathrm{CTb}$ into the DVC on P6 consistently produced retrograde labeling of many neurons in both the IC and infralimbic mPFC (Fig. 7), as well as neurons in the LHA, PVN, CeA, and BNST. Few retrogradely labeled DMH neurons were observed after injection of $\mathrm{CTb}$ into the DVC on P6, similar to the results obtained in rats injected with $\mathrm{CTb}$ on P1.

Many retrogradely labeled neurons were present in layer $\mathrm{V}$ of the primary motor cortex in the P6-P8 group, likely a result of tracer diffusion from the $\mathrm{CTb}$ injection site into the subjacent hypoglossal motor nucleus and/or adjacent dorsal reticular formation. Similar tracer spread also occurred in some of the rats injected on P1 (see Fig. 6), but in those cases cortex labeling was not observed.

\section{DISCUSSION}

Results from this study demonstrate a novel anatomical strategy for evaluating synaptic connectivity in developing neural circuits and show a temporally segregated postnatal emergence of medial hypothalamic, lateral hypothalamic, and telencephalic synaptic inputs to central autonomic and preautonomic neurons in rats.
Stomach wall PRV inoculation parameters and postinjection survival times were kept constant and generated similar patterns of neuronal infection in the spinal cord and caudal brainstem of rats in each age group. Autonomic control regions of the diencephalon (DMH, LHA, and PVN) and telencephalon (CeA, BNST, IC, and $\mathrm{mPFC}$ ) were transneuronally infected after stomach wall inoculation in the P8-P10.5 age group, as in adult rats (Rinaman et al., 1993; Yang et al., 1999). However, the visceral cortex (IC and $\mathrm{mPFC}$ ) was not infected after stomach wall inoculation of PRV in the P4-P6.5 age group, and the caudal LHA, CeA, BNST, IC, and mPFC were not infected after stomach wall inoculation in the P1-P3.5 age group. Thus, diencephalic and telencephalic labeling increased with postnatal age, despite the fact that in older rats the virus had to be transported a greater distance in the same 62-64 hr postinoculation survival interval. These results provide evidence of progressive increases in synaptic connectivity between limbic forebrain neurons and autonomic neurons in rats postnatally and demonstrate the efficacy of using the PRV-tracing method to monitor temporal differences in the development of axonal projections and synaptic connections between functionally related CNS regions. 


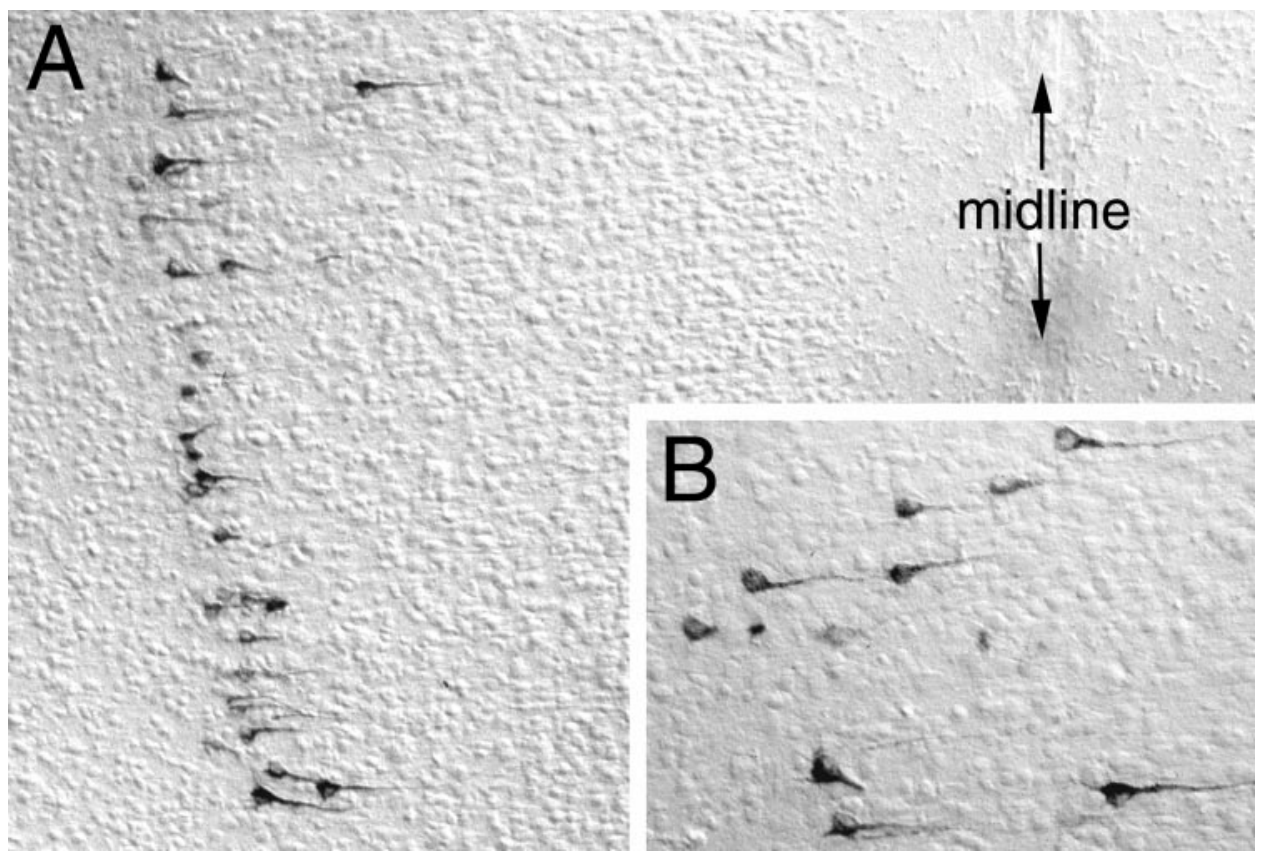

Figure 7. Photomicrographs of CTb immunolabeling in the mPFC $48-50 \mathrm{hr}$ after injection of CTb directly into the DVC on P6. Labeled neurons visible near the top of the field in $A$ are shown at higher magnification in $B$.

\section{Specificity of PRV as a transneuronal tracer}

PRV exhibits a pronounced affinity for axon terminals (Marchand and Schwab, 1987) that results in its uptake by neurons after peripheral or central inoculation. This initial invasive event is followed by retrograde transport, replication, and transneuronal passage of PRV through central polysynaptic circuits (cf. Enquist et al., 1999). All strains of PRV that have been used for neural tract tracing, including the attenuated vaccine strain (Bartha) used in the present study, ultimately can produce neuronal pathology and necrosis after prolonged intracellular replication. Importantly, however, the potential nonspecific extracellular spread of PRV from pathologically infected neurons is restricted by glia, macrophages, and other immune cells that are recruited to sites of neuronal infection (Card et al., 1990, 1993; Rinaman et al., 1993; Rassnick et al., 1998). These reactive cells effectively isolate infected neurons and contribute to the removal of cellular debris, thereby contributing to the demonstrated transynaptic specificity of PRV transport in adult rats (Card et al., 1993; Rinaman et al., 1993). In consequence, nonspecific central neuronal infection is not observed, even in brain regions that are juxtaposed directly to those containing severely infected necrotic neurons. We reported recently that spatiotemporally ordered astrocytic and microglial responses to central PRV infection in 1-d-old rats are similar to responses in adult rats (Rinaman et al., 1999). In that study and in the present one, central neuronal infection was observed exclusively in autonomic-related regions of the CNS after injection of PRV into the ventral stomach wall. These data together indicate that the PRV-tracing method is a sensitive and suitable approach for investigating synaptically linked circuits in the mature and developing CNS. It is important to note, however, that the ability of newly developed synapses to transport PRV has not yet been temporally correlated with the emergence of "functional connectivity" in terms of presynaptic neurotransmitter release and postsynaptic sensitivity.

\section{Parasympathetic contribution to transneuronal telencephalic labeling}

A control experiment was performed in the present study, in which PRV was injected into the abdominal muscles overlying the stomach in rats on P8. This experiment was performed because inoculation of the ventral stomach wall required passing a PRVfilled glass micropipette through those abdominal muscles. The resulting pattern of transneuronal CNS infection was very different from the pattern observed in rats of the same age after inoculation of the stomach wall. Spinal cord, brainstem, and cerebellar infection in muscle-injected rats was consistent with retrograde transneuronal transport of PRV through both sympathetic (vascular) and somatic neural circuits; vagal parasympathetic motor neurons in the DVC were not infected. Transneuronally infected forebrain neurons were prominent in the caudal LHA, DMH, dorsal and medial parvocellular PVN, primary motor cortex (layer $\mathrm{V}$ pyramidal neurons), and supplemental somatosensory cortex after abdominal muscle inoculation. However, infected neurons were absent in the CeA, BNST, IC, and $\mathrm{mPFC}$, despite robust infection of sympathetic and presympathetic neurons in the spinal cord, brainstem, and hypothalamus. Thus, transneuronal infection of telencephalic neurons after stomach wall inoculation likely occurred through projections to the parasympathetic DVC. By the same logic, transneuronal infection of hypothalamic neurons could have occurred through both sympathetic and parasympathetic routes. Other data consistent with these conclusions were obtained in adult rats in which PRV was injected into the pancreas after sympathetic or parasympathetic routes of forebrain infection were surgically eliminated (Loewy and Haxhiu, 1993; Jansen et al., 1997). Studies using conventional anterograde and retrograde tract tracers also have shown that the LHA and PVN project directly to both sympathetic and parasympathetic preganglionic neurons in adult rats, whereas autonomic-related regions of the telencephalon preferentially target the DVC (Loewy, 1991).

\section{Age-related increases in transneuronal forebrain labeling after stomach wall inoculation}

The absence of PRV labeling in the LHA, CeA, and BNST in the P1-P3.5 age group is not caused by an inability of neurons in those areas to take up or replicate virus. Many LHA, CeA, and BNST neurons were retrogradely labeled after injection of PRV directly into the DVC on $\mathrm{P} 1$, indicating that these forebrain 
neurons already have axons in or near the DVC and that the cellular mechanisms necessary for PRV neuronal invasion, retrograde transport, and replication already are functional. Together the data suggest either that the axons of LHA, CeA, and BNST neurons do not yet form synaptic contacts with gastric-related DVC neurons in rats on P1-P3.5 or that the number of such synaptic contacts is insufficient to achieve productive transneuronal retrograde PRV infection (for discussion of this general issue, see Card et al., 1995, 1999). By extension, cortical neurons do not have axons in or near the DVC in rats on P1, because no cortical labeling was observed 48-50 hr after DVC injections of either $\mathrm{PRV}$ or CTb on P1. However, injections of CTb into the DVC on $\mathrm{P} 6$ produced retrograde labeling in both the IC and $\mathrm{mPFC}$, as in adult rats, indicating that initial visceral cortex axonal projections to the DVC appear sometime between P1 and P6.

\section{Functional role of limbic-autonomic circuits}

Autonomic responses to stimuli that elicit fear, anxiety, or other strong emotions typically include significant alterations of cardiovascular and gastrointestinal functions. The hypothalamus, CeA, BNST, IC, and mPFC serve as principal gateways for septohippocampal and cortical influences over autonomic response components of anxiety and contextual fear conditioning (Pratt, 1992; Phillips and LeDoux, 1994; Stein, 1998; Vouimba et al., 1998; Crestani et al., 1999). Results from the present study indicate that the first $10 \mathrm{~d}$ of rat postnatal development are characterized by significant, temporally sequenced synapse formation occurring between neurons in these diencephalic and telencephalic regions and autonomic circuits in the brainstem and spinal cord. The functional implications of this developmental sequence are unknown. By analogy to other CNS systems, one might hypothesize that this early postnatal period represents a "critical period" of limbic-autonomic circuit development, during which time experience or environmental events might participate in shaping ongoing synapse formation. Although this remains speculative, it is appropriate to consider what is known about the structural and functional links between the limbic forebrain and autonomic neurons, as briefly summarized below.

\section{Dorsomedial nucleus of the hypothalamus}

The DMH is implicated in visceral reactions to stress and anxiety (cf. Thompson et al., 1996). In the present study, DMH neurons consistently were labeled at all ages examined after injection of PRV either into the stomach wall or directly into the DVC. By comparison, DMH neurons were much less frequently labeled after injection of $\mathrm{CTb}$ into the DVC on P1 or P6. The CTbtracing results are consistent with previous studies indicating that the DMH projects mainly to intrahypothalamic targets and sends a relatively sparse direct projection to the DVC (Thompson et al., 1996). Considered together, these findings support the view that the more robust DMH labeling observed after PRV injections into the stomach wall or DVC is the result of transneuronal transport through another infected cell group, probably the PVN and/or LHA. Particularly dense DMH projections terminate in dorsal, ventral, and perifornical parvocellular subnuclei of the PVN and in the caudal LHA, which contain neurons that project directly to autonomic brainstem and spinal cord neurons in adult rats (Saper et al., 1976). The transneuronal DMH labeling observed after injection of PRV into the ventral stomach wall on P1 likely originated from DMH projections to infected PVN neurons rather than from DMH projections to the LHA, because LHA neurons were not labeled in this experimental group. The increased amount of transneuronal DMH labeling observed after injection of PRV into the ventral stomach wall on P4 or P8, or after injection of PRV into the DVC on P1 or P6, could have originated from both the PVN and the LHA, because both areas contained many infected neurons in those experimental groups.

\section{Lateral hypothalamic area}

Electrolytic or cytotoxic lesions of the LHA do not affect autonomic responses to unconditioned (e.g., nociceptive) stimuli but significantly attenuate the expression of associative (learned) sympathetic and parasympathetic responses in rabbit, rat, and monkey (Smith et al., 1980; Francis et al., 1981; LeDoux et al., 1988). LHA neurons project to both the DVC and the intermediolateral cell column of the thoracolumbar spinal cord in adult rats (Saper et al., 1976). An important role for LHA projections to the DVC in gastrointestinal functions has been suggested on the basis of LHA involvement in cephalic-phase (conditioned) gastric secretion and stress-related gastric and duodenal mucosal erosion (Landeira-Fernandez and Grijalva, 1999; Takahashi et al., 1999). The existence of axonal projections from LHA neurons to the region of the DVC in neonatal rats was demonstrated in the present study after injection of CTb into the DVC on P1 or P6. However, the caudal LHA neurons that are retrogradely labeled on P1 appear not to form synaptic contacts with gastricrelated DVC neurons, as evidenced by the lack of transneuronal labeling in the caudal LHA after injection of PRV into the ventral stomach wall on P1. The transneuronal LHA labeling observed after PRV inoculation of the stomach wall on P4 or P8, as in adult rats, indicates a gradual assembly of this functional circuit.

\section{Paraventricular nucleus of the hypothalamus}

Parvocellular PVN neurons project directly to sympathetic and parasympathetic preganglionic neurons in the spinal cord and DVC (Swanson and Kuypers, 1980) and contribute importantly to the central regulation of gastric and other autonomic functions (Rogers and Hermann, 1992; Sawchenko et al., 1996). PVN neurons that project to the DVC and contain corticotropinreleasing factor have been implicated in the production of several stress-related gastric effects, including increased acid secretion, inhibition of emptying, and gastroduodenal erosions (Ferguson et al., 1988; Tache et al., 1999). Our results indicate that PVN synaptic projections to gastric-related autonomic neurons are present as early as P1, consistent with a previous report (Rinaman et al., 1999). The present study extends those results by demonstrating that the number of transneuronally infected PVN neurons increases in rats during the first $10 \mathrm{~d}$ of postnatal development, suggesting that hypothalamic control over gastric function undergoes a significant amount of early postnatal maturation.

Substantial postnatal maturation of PVN oxytocinergic inputs to the DVC also has been demonstrated (Rinaman, 1998). This is especially relevant to the present study, because oxytocinergic projections seem to be critical for PVN control over gastric function (Rogers and Hermann, 1985, 1987, 1992). It is interesting to note that the pattern of gastric changes elicited by microinjection of oxytocin into the DVC mimics the changes that occur at the first taste of an anticipated meal, even before any food is swallowed (Rogers and Hermann, 1987, 1992; Rogers et al., 1995). As suggested by Rogers and Hermann (1992), CNS regions that are involved in interpreting external sensory events linked to the arrival of food might use the PVN-DVC oxytocinergic pathway as a conduit to evoke the appropriate anticipatory changes in gastrointestinal function. 


\section{Central nucleus of the amygdala and bed nucleus of the stria terminalis}

Detailed anatomical studies in rats have revealed that the parvocellular PVN, CeA, and BNST together form a reciprocally interconnected continuum of "prevagal" neurons (Schwaber et al., 1982). Our results indicate that only a subset of the PVN component of this prevagal group innervates gastric-related DVC neurons in rats between $\mathrm{P} 1$ and $\mathrm{P} 3.5$, with additional $\mathrm{PVN}, \mathrm{CeA}$, and BNST prevagal components emerging during the subsequent week. The CeA and BNST together have been implicated in the expression of autonomic correlates of emotional arousal (cf. LeDoux et al., 1988). Direct descending projections from the CeA and BNST to the DVC (Schwaber et al., 1982; Van der Kooy et al., 1984; Danielsen et al., 1989) represent a sufficient pathway for the regulation of autonomic state by telencephalic structures (cf. Bernston et al., 1998). Results from anatomical and electrophysiological studies suggest that visceral activation accompanying emotional responses involves direct inhibition of GABAergic preautonomic DVC neurons by neurons in the medial $\mathrm{CeA}$, at least a portion of which are somatostatinergic (Rogers and Hermann, 1992; Pickel et al., 1996). Thus, stimulation of inhibitory CeA inputs to the DVC should produce a disinhibition of vagal motor outflow, consistent with evidence that electrical or chemical stimulation of either the CeA or BNST increases vagally mediated gastric motility in adult rats (Hermann et al., 1990). Our present findings suggest that the CeA and BNST do not directly modulate vagal motor function in rats until at least several days after birth.

\section{Insular cortex and medial prefrontal cortex}

The DVC receives direct input from pyramidal neurons in the IC and mPFC (Saper, 1982; Terreberry and Neafsay, 1983; Van der Kooy et al., 1984; Yasui et al., 1991b). These "visceral cortex" areas also project to autonomic regions of the PVN, CeA, and BNST and have been implicated in autonomic reactivity associated with anxiety and other affective processes (LeDoux et al., 1988). Stimulation of the IC or mPFC potently alters autonomic outflow, producing an inhibition of gastrointestinal activity similar to the inhibition produced by exposure to fear-inducing stimuli (Yasui et al., 1991a; Aleksandrov et al., 1996). Results from the present study indicate that gastric-related autonomic neurons receive demonstrable synaptic inputs from neurons in the IC and mPFC by P10.5, but not at P8.5 or before. The implication of these results is that the visceral cortex does not directly modulate autonomic function in rats until after the first week of postnatal development. In fact, the axons of IC and mPFC neurons appear not to even reach the vicinity of the DVC until at least 2 or more days after birth, because neither IC nor mPFC neurons were retrogradely labeled after tracer injections (PRV or $\mathrm{CTb}$ ) were made into the DVC on P1. Similarly, neurons in the primary somatic motor cortex were not retrogradely labeled after CTb tracer injections that involved the hypoglossal motor nucleus and adjacent dorsal reticular formation on P1, although similar tracer injections made on P6 did produce retrograde labeling of neurons in both the visceral and somatic motor cortices. These findings are consistent with previous reports that corticospinal fibers first reach the cervical spinal cord (just caudal to the DVC) in rats on $\mathrm{P} 1$, although there is a $2 \mathrm{~d}$ delay between the arrival of corticospinal fibers in the ventral and lateral funiculi and their invasion of the ventral horn gray matter at the same spinal level (cf. Lakke, 1997).

\section{Summary and conclusions}

Emotional and other cognitive experiences clearly are associated with autonomic responses in humans and other animals, including rats. Exaggerated visceral reactivity in humans is recognized as a common symptom of anxiety disorders, which feature inappropriate autonomic responses to certain triggering stimuli. The long-lasting influence of early epigenetic events on later behavioral and physiological responses to emotional stimuli (Jordan, 1990) presumably is linked to altered formation of the limbicautonomic circuits that underlie affective expression. This hypothesis has been difficult to evaluate experimentally, however, because the normal developmental assembly of these neural circuits has not been determined. Results from the present study provide the first evidence that autonomic neurons in rats are not subject to significant direct descending inputs from most regions of the limbic forebrain until several days after birth, whereas a subset of medial hypothalamic neurons appear to innervate gastric-related autonomic neurons as early as P3. The data also demonstrate the potentially broad applicability of viral-tracing methods to decipher complex events in circuit formation during brain development.

\section{REFERENCES}

Abercrombie M (1946) Estimation of nuclear populations from microtome sections. Anat Rec 94:239-247.

Aleksandrov VG, Bagaev VA, Nozdrachev AD, Panteleev SS (1996) Identification of gastric related neurones in the rat insular cortex. Neurosci Lett 216:5-8.

Bartha A (1961) Experimental reduction of virulence of Aujeszky's disease virus. Magy Allotorv Lapja 16:42-45.

Bernston GG, Sarter M, Cacioppo JT (1998) Anxiety and cardiovascular reactivity: the basal forebrain cholinergic link. Behav Brain Res 94:225-248.

Card JP (1995) Pseudorabies virus replication and assembly in the rodent central nervous system. In: Viral vectors (Kaplitt MG, Loewy AD, eds), pp 319-347. Orlando, FL: Academic.

Card JP (1998) Practical considerations for the use of pseudorabies virus in transneuronal studies of neural circuitry. Neurosci Biobehav Rev 22:685-694.

Card JP, Enquist LW (1995) Neurovirulence of pseudorabies virus. Crit Rev Neurobiol 9:137-162.

Card JP, Rinaman L, Schwaber JS, Miselis RR, Whealy ME, Robbins AK, Enquist LW (1990) Neurotropic properties of pseudorabies virus: uptake and transneuronal passage in the rat central nervous system. J Neurosci 10:1974-1994.

Card JP, Rinaman L, Lynn RB, Lee B-H, Meade RP, Miselis RR, Enquist LW (1993) Pseudorabies virus infection of the rat central nervous system: ultrastructural characterization of viral replication, transport, and pathogenesis. J Neurosci 13:2515-2539.

Card JP, Dubin JR, Whealy ME, Enquist LW (1995) Influence of infectious dose upon productive replication and transynaptic passage of pseudorabies virus in rat central nervous system. J Neurovirol $1: 349-358$.

Card JP, Enquist LW, Moore RY (1999) Neuroinvasiveness of pseudorabies virus injected intracerebrally is dependent on viral concentration and terminal field density. J Comp Neurol 407:438-452.

Crestani F, Lorez M, Baer K, Essrich C, Benke D, Laurent JP, Belzung C, Fritschy JM, Luscher B, Mohler H (1999) Decreased GABAAreceptor clustering results in enhanced anxiety and a bias for threat cues. Nat Neurosci 2:833-839.

Cunningham MG, McKay RDG (1993) A hypothermic miniaturized stereotaxic instrument for surgery in newborn rats. J Neurosci Methods 47:105-114.

Danielsen EH, Magnuson DJ, Gray TS (1989) The central amygdaloid nucleus innervation of the dorsal vagal complex in rat: a Phaseolus vulgaris leucoagglutinin lectin anterograde tracing study. Brain Res Bull 22:705-715.

Enquist LW, Card JP (1996) Pseudorabies virus: a tool for tracing neuronal connections. In: Protocols for gene transfer in neuroscience: 
towards gene therapy of neurological disorders (Lowenstein PR, Enquist LW, eds), pp 333-348. Chichester, UK: Wiley.

Enquist LW, Husak PJ, Banfield BW, Smith GA (1999) Infection and spread of alphaherpesviruses in the nervous system. Adv Virus Res 51:237-347.

Ferguson AV, Marcus P, Spencer J, Wallace JL (1988) Paraventricular nucleus stimulation causes gastroduodenal mucosal necrosis in the rat. Am J Physiol 255:R861-R865.

Francis J, Hernandez LL, Powell DA (1981) Lateral hypothalamic lesions: effects on Pavlovian cardiac and eyeblink conditioning in the rabbit. Brain Res Bull 6:155-163.

Gray TS, Magnuson DJ (1987) Neuropeptide neuronal efferents from the bed nucleus of the stria terminalis and central amygdaloid nucleus to the dorsal vagal complex in the rat. J Comp Neurol 262:365-374.

Hermann GE, McCann MJ, Rogers RC (1990) Activation of the bed nucleus of the stria terminalis increases gastric motility in the rat. $\mathrm{J}$ Auton Nerv Syst 30:123-128.

Jansen ASP, Hoffman JL, Loewy AD (1997) CNS sites involved in sympathetic and parasympathetic control of the pancreas: a viral tracing study. Brain Res 766:29-38.

Jordan D (1990) Autonomic changes in affective behavior. In: Central regulation of autonomic functions (Loewy AD, Spyer KM, eds), pp 349-365. New York: Oxford UP.

Lakke EA (1997) The projections to the spinal cord of the rat during development: a timetable of descent. Adv Anat Embryol Cell Biol 135:1-143.

Lakke EA, Hinderink JB (1989) Development of the spinal projections of the nucleus paraventricularis hypothalami of the rat: an intra-uterine WGA-HRP study. Dev Brain Res 49:115-121.

Landeira-Fernandez J, Grijalva CV (1999) Gastric mucosal erosion produced by NMDA microinfusions in the lateral hypothalamus: effect of selective knife cuts. Behav Brain Res 102:51-60.

LeDoux JE, Iwata J, Cicchetti P, Reis DJ (1988) Different projections of the central amygdaloid nucleus mediate autonomic and behavioral correlates of conditioned fear. J Neurosci 8:2517-2529.

Loewy AD (1991) Forebrain nuclei involved in autonomic control. Prog Brain Res 87:253-268.

Loewy AD, Haxhiu MA (1993) CNS cell groups projecting to pancreatic parasympathetic preganglionic neurons. Brain Res 620:323-330.

Loewy AD, Spyer KM, eds (1990) Central regulation of autonomic functions. New York: Oxford UP.

Marchand CF, Schwab ME (1987) Binding, uptake and retrograde axonal transport of herpes virus suis in sympathetic neurons. Brain Res 383:262-270

McLean IW, Nakane PK (1974) Periodate-lysine-paraformldehyde fixative. A new fixative for immunoelectron microscopy. J Histochem Cytochem 22:1077-1083.

Miller AJ, McKoon M, Pinneau M, Silverstein R (1983) Postnatal synaptic development of the nucleus tractus solitarius (NTS) of the rat. Dev Brain Res 8:205-213.

Phillips RG, LeDoux JE (1994) Lesions of the dorsal hippocampal formation interfere with background but not foreground contextual fear conditioning. Learn Mem 1:34-44.

Pickel VM, Bockstaele EJV, Chan J, Cestari DM (1996) GABAergic neurons in rat nuclei of solitary tracts receive inhibitory-type synapses from amygdaloid efferents lacking detectable GABA-immunoreactivity. J Neurosci Res 44:446-458.

Pratt JA (1992) The neuroanatomical basis of anxiety. Pharmacol Ther 55:149-181.

Rao H, Pio J, Kessler JP (1999) Postnatal development of synaptophysin immunoreactivity in the rat nucleus tractus solitarii and caudal ventrolateral medulla. Dev Brain Res 112:281-285.

Rassnick S, Enquist LW, Sved AF, Card JP (1998) Pseudorabies virusinduced leukocyte trafficking into the rat central nervous system. J Virol 72:9181-9191.

Rinaman L (1998) Oxytocinergic inputs to the nucleus of the solitary tract and dorsal motor nucleus of the vagus in neonatal rats. J Comp Neurol 399:101-109.

Rinaman L, Levitt P (1993) Establishment of vagal sensory-motor circuits during fetal development in rats. J Neurobiol 24:641-659.

Rinaman L, Card JP, Schwaber JS, Miselis RR (1989) Ultrastructural demonstration of a gastric monosynaptic vagal circuit in the nucleus of the solitary tract in rat. J Neurosci 9:1985-1996.

Rinaman L, Card JP, Enquist LW (1993) Spatiotemporal responses of astrocytes, ramified microglia, and brain macrophages to central neuronal infection with pseudorabies virus. J Neurosci 13:685-702.

Rinaman L, Roesch MR, Card JP (1999) Retrograde transynaptic pseudorabies virus infection of central autonomic circuits in neonatal rats. Dev Brain Res 114:207-216.

Rogers RC, Hermann GE (1985) Dorsal medullary oxytocin, vasopressin, oxytocin antagonist, and TRH effects on gastric acid secretion and heart rate. Peptides 6:1143-1148.

Rogers RC, Hermann GE (1987) Oxytocin, oxytocin antagonist, TRH, and hypothalamic paraventricular nucleus stimulation effects on gastric motility. Peptides 8:505-513.

Rogers RC, Hermann GE (1992) Central regulation of brainstem gastric vago-vagal control circuits. In: Neuroanatomy and physiology of abdominal vagal afferents (Ritter S, Ritter RC, Barnes CD, eds), pp 99-134. Boca Raton, FL: CRC.

Rogers RC, McTigue DM, Hermann GE (1995) Vagovagal reflex control of digestion: afferent modulation by neural and "endoneurocrine" factors. Am J Physiol 268:G1-G10.

Saper CB (1982) Convergence of autonomic and limbic connections in the insular cortex of the rat. J Comp Neurol 210:163-173.

Saper CB, Loewy AD, Swanson LW, Cowan WM (1976) Direct hypothalamo-autonomic connections. Brain Res 117:305-312.

Sawchenko PE, Brown ER, Chan RKW, Ericsson A, Li H-Y, Roland BL, Kovacs KJ (1996) The paraventricular nucleus of the hypothalamus and the functional neuroanatomy of visceromotor responses to stress. Prog Brain Res 107:201-222.

Schwaber JS, Kapp BS, Higgins GA, Rapp PR (1982) Amygdaloid and basal forebrain connections with the nucleus of the solitary tract and the dorsal motor nucleus. J Neurosci 2:1424-1438.

Shapiro RE, Miselis RR (1985) The central organization of the vagus nerve innervating the stomach of the rat. J Comp Neurol 238:473-488.

Smith OA, Astley CA, DeVito JL, Stein JM, Walsh RE (1980) Functional analysis of hypothalamic control of the cardiovascular responses accompanying emotional behavior. Fed Proc 29:2487-2494.

Stein MB (1998) Neurobiological perspectives on social phobia: from affiliation to zoology. Biol Psychiatry 44:1277-1285.

Swanson LW (1992) Brain maps. Structure of the rat brain. Amsterdam: Elsevier.

Swanson LW, Kuypers HGJM (1980) The paraventricular nucleus of the hypothalamus: cytoarchitectonic subdivisions and the organization of projections to the pituitary, dorsal vagal complex, and spinal cord as demonstrated by retrograde fluorescence double-labeling methods. J Comp Neurol 194:555-570.

Tache Y, Martinez V, Million M, Rivier J (1999) Corticotropinreleasing factor and the brain-gut motor response to stress. Can $\mathrm{J}$ Gastroenterol 13[Suppl A]:A18-A25.

Takahashi N, Okumura T, Yamada H, Kohgo Y (1999) Stimulation of gastric acid secretion by centrally administered orexin-A in conscious rats. Biochem Biophys Res Commun 254:623-627.

Terreberry RR, Neafsay EJ (1983) Rat medial frontal cortex: a visceral motor region with a direct projection to the solitary nucleus. Brain Res 278:245-249.

Thompson RH, Canteras NS, Swanson LW (1996) Organization of projections from the dorsomedial nucleus of the hypothalamus: a PHA-L study in the rat. J Comp Neurol 376:143-173.

Van der Kooy D, Koda LK, McGinty JF, Gerfen CR, Bloom FE (1984) The organization of projections from the cortex, amygdala, and hypothalamus to the nucleus of the solitary tract in rat. J Comp Neurol 1984:1-24.

Vouimba RM, Garcia R, Jaffard R (1998) Opposite effects of lateral septal LTP and lateral septal lesions on contextual fear conditioning in mice. Behav Neurosci 112:875-884.

Watson RE, Wiegand ST, Clough RW, Hoffman GE (1986) Use of cryoprotectant to maintain long-term peptide immunoreactivity and tissue morphology. Peptides 7:155-159.

Yang M, Card JP, Tirabassi RS, Miselis RR, Enquist LW (1999) Retrograde, transneuronal spread of pseudorabies virus in defined neuronal circuitry of the rat brain is facilitated by $\mathrm{gE}$ mutations that reduce virulence. J Virol 73:4350-4359.

Yasui Y, Breder CD, Saper CB, Cechetto DF (1991a) Autonomic responses and efferent pathways from the insular cortex in the rat. J Comp Neurol 303:355-374.

Yasui Y, Itoh K, Kaneko T, Shigemoto R, Mizuno N (1991b) Topographical projections from the cerebral cortex to the nucleus of the solitary tract in the cat. Exp Brain Res 85:75-84. 